\title{
The effect of reproductive performance on the dairy cattle herd value assessed by integrating a daily dynamic programming model with a daily Markov chain model
}

\author{
A. S. Kalantari and V. E. Cabrera ${ }^{1}$ \\ Department of Dairy Science, University of Wisconsin-Madison, Madison 53706-1284
}

\begin{abstract}
The objective of this study was to determine the effect of reproductive performance on dairy cattle herd value. Herd value was defined as the herd's average retention payoff (RPO). Individual cow RPO is the expected profit from keeping the cow compared with immediate replacement. First, a daily dynamic programming model was developed to calculate the RPO of all cow states in a herd. Second, a daily Markov chain model was applied to estimate the herd demographics. Finally, the herd value was calculated by aggregating the RPO of all cows in the herd. Cow states were described by 5 milk yield classes $(76,88$, 100,112 , and $124 \%$ with respect to the average), 9 lactations, $750 \mathrm{~d}$ in milk, and $282 \mathrm{~d}$ in pregnancy. Five different reproductive programs were studied (RP1 to RP5). Reproductive program 1 used 100\% timed artificial insemination (TAI; $42 \%$ conception rate for first TAI and 30\% for second and later services) and the other programs combined TAI with estrus detection. The proportion of cows receiving artificial insemination after estrus detection ranged from 30 to $80 \%$, and conception rate ranged from 25 to $35 \%$. These 5 reproductive programs were categorized according to their $21-\mathrm{d}$ pregnancy rate (21-d PR), which is an indication of the rate that eligible cows become pregnant every $21 \mathrm{~d}$. The 21 -d PR was $17 \%$ for RP1, $14 \%$ for RP2, $16 \%$ for RP3, $18 \%$ for RP4, and 20\% for RP5. Results showed a positive relationship between 21-d PR and herd value. The most extreme herd value difference between 2 reproductive programs was $\$ 77 /$ cow per yr for average milk yield (RP5 - RP2), $\$ 13 /$ cow per yr for lowest milk yield (RP5 - RP1), and $\$ 160 /$ cow per yr for highest milk yield (RP5 - RP2). Reproductive programs were ranked based on their calculated herd value. With the exception of the best reproductive program (RP5), all
\end{abstract}

Received March 30, 2012.

Accepted June 26, 2012.

${ }^{1}$ Corresponding author: vcabrera@wisc.edu other programs showed some level of ranking change according to milk yield. The most dramatic ranking change was observed in RP1, which moved from being the worst ranked for lowest milk yield to the second ranked for highest milk yield. Within a reproductive program, RPO changed based on the stage of lactation at pregnancy. Cows getting pregnant in the early stage of a lactation had higher RPO compared with getting pregnant later in the lactation. However, the RPO at calving was similar for early and late lactation pregnancies.

Key words: retention payoff, replacement decision, optimization, simulation

\section{INTRODUCTION}

Reproductive performance affects dairy herd profitability (Britt, 1985; Meadows et al., 2005; Olynk and Wolf, 2008). The association between reproductive performance and profitability is a result of effects on milk yields, available replacement heifers, and voluntary and involuntary culling rates (Olynk and Wolf, 2008).

Some studies that have evaluated the economic impact of different reproductive programs using simulation models (Olynk and Wolf, 2009; Cabrera and Giordano, 2010; Giordano et al., 2011a,b) focused on the impact of reproductive programs alone. However, replacement decisions also greatly affect a herd's profitability (van Arendonk, 1985).

Several biological and economic factors should be considered in order to make optimal replacement decisions. The most important factors are milk production, pregnancy, stage of lactation, and the value of a replacement heifer. Dynamic programming (DP), also known as Markov decision process (MDP), is an optimization technique that can handle all of these factors. Dynamic programming models have been developed to optimize culling decisions in dairy herds over the past several decades (Smith, 1973; van Arendonk, 1985; Kristensen, 1988; De Vries, 2004). The stage length of the DP models in these studies varied from 1 yr to 1 mo. However, farmers are making these decisions on 
a daily basis. Thus, the applicability of these models for practical farm decision making has been limited (Nielsen et al., 2010).

Recently, Nielsen et al. (2010) used a daily stage length on DP to find the optimal replacement policy in dairy herds. They used a hierarchical MDP algorithm, developed by Kristensen (1988). Emphasis in that study was given to building a DP model to use daily milk yield performances based on the modern milking system in Denmark (Nielsen et al., 2010). Nonetheless, the Nielsen et al. (2010) study did not address the effect of different reproductive programs on replacement decisions and retention payoff (RPO, the expected profit from keeping the cow compared with immediate replacement). Hence, the need still exists to evaluate the combined effect of reproductive performance and optimal replacement decisions. A Markov chain simulation model could be a useful technique to approximate the herd structure (De Vries, 2004) used to calculate the weighted average RPO after determining the optimal decisions for all cow states with a DP model.

The main goal of this study was thus to assess the economic impact of reproductive performance under optimal replacement policies. The specific objectives were (1) to develop a daily DP model to compare different reproductive programs' herd values by using the herd structure found with a daily Markov chain model, and (2) to show the effect of pregnancy time on the $\mathrm{RPO}$ within specific reproductive programs.

\section{MATERIALS AND METHODS}

A value iteration method (De Vries, 2004) was used in this study to find the optimum replacement policies of the DP problem. After optimizing replacement decisions, a Markov chain model was applied to simulate the herd structure over time (De Vries, 2004; Cabrera, 2010; Kalantari et al., 2010). A daily Markov chain model developed by Giordano et al. (2012) was used to correspond to the dimensions of a daily DP model. Finally, a herd value was calculated by multiplying the RPO resulting from the DP model by the proportion of cows from the Markov chain results. This herd value thus implicitly captures all optimal replacement decisions.

\section{DP Model}

Four state variables were included to describe cows in the DP model. Cow state was defined by milk class ( $\boldsymbol{c}=1$ to 5$)$, lactation number $(\boldsymbol{l}=1$ to 9$)$, DIM ( $\boldsymbol{d}=$ 1 to 750 ), and days in pregnancy (DIP; $\boldsymbol{p}=0$ to 282 ). Multiplying all these dimensions creates over 9 million total cow states, but not all of these states are possible because of biological or imposed constraints. Biological constraints indicate that DIP is always greater than DIM. Imposed constraints determine a voluntary waiting period (VWP) and last DIM for breeding services. After excluding impossible states, the total number of possible states in the calculations was $>3$ million. For each state variable, several stochastic elements or transition probabilities are included in the model, such as the probability of abortion, pregnancy, or involuntary culling, and the probability of transition to different milk classes. All of these transition probabilities were accordingly defined on a daily basis.

The daily DP model presented here was developed following the monthly model developed by De Vries (2004). However, in the current daily model, the stage variable was deleted from the dimensions of some of the equations. The reason for doing this was to avoid the out-of-memory exception error in the Windows platform due to the large dimension of the model with daily stages. With this modification, the very large problem was built as a backward induction (value iteration) method without affecting the results. Other modification to the De Vries (2004) formulation was the inclusion of the transition probabilities of abortion (De Vries, 2006).

The expected net present value (NPV) of the cash flow $\left(F_{c, l, d, p}\right)$ under an optimum policy was

$$
F_{c, l, d, p}=\operatorname{Max}\left[\operatorname{Keep}_{c, l, d, p}, \operatorname{Repl}_{c, l, d, p}\right],
$$

where $K e e p_{c, l, d, p}=$ expected NPV of keeping the cow given the optimal decisions in the remainder stages, and $R e p l_{c, l, d, p}=$ expected NPV of replacing the cow given the optimal decisions in the remainder stages:

$$
\begin{gathered}
\operatorname{Repl}_{c, l, d, p}=S E L L_{l, d}+F H_{t}, \text { and } \\
F H_{t}=-C+\delta \sum_{c=1}^{5} P H(c)\left\{\begin{array}{l}
R E V_{c, 1,1,0}+\left[1-\operatorname{Pinv}_{1,1}\right] \times F_{c, 1,2,0} \\
+\operatorname{Pinv} v_{1,1} \times\left[S E L L_{1,2}+F H_{t+1}\right]
\end{array}\right\},
\end{gathered}
$$

where $S E L L_{l, d}=$ carcass value; $F H_{t}$ expected NPV of cash flow for a replacement heifer entering at stage $t$; $C=$ cost of replacement heifer; $P H(c)=$ probability of replacement heifer with production level $c ; R E V_{c, l, d, p}=$ net revenue for current state; $\operatorname{Pinv}_{l, d}=$ probability of involuntary culling at each day; and $\delta=$ discount factor.

The expected NPV for the keep decision depends on the state of the cow. Following are the keep value calculation formulas: 
1) If the cow was eligible for insemination $(p=0$ and VWP $\leq d \leq 300$ ), then the keep value depended on the insemination cost (PregCost):

$$
\begin{aligned}
& \operatorname{Keep}_{c, l, d, 0}=R E V_{c, l, d, 0}+\text { PregCost }_{d}
\end{aligned}
$$

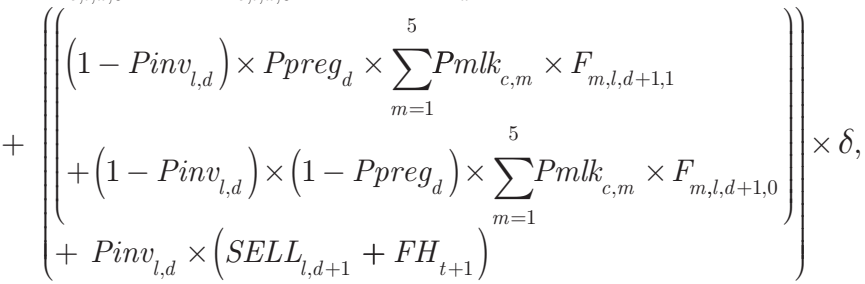

where Ppreg $_{d}=$ daily probability of pregnancy, and $P m l k_{c, m}=$ daily probability of changing among milk classes.

$2)$ If the cow was open in their last possible DIM ( $p$ $=0$ and $d=750)$ or if the cow was in the last day in pregnancy and last possible lactation $(p=$ 282 and $l=9$ ), then

$$
K e e p_{c, l, d, p}=R E V_{c, l, d, p}+\left(S E L L_{l, d+1}+F H_{t+1}\right) \times \delta .
$$

3) If the cow was open and not eligible for insemination $(p=0$ and $d<\operatorname{VWP}$ or $300 \leq d \leq 749)$ or if the cow was pregnant $(1 \leq p \leq 281)$, then

$$
\begin{aligned}
& \operatorname{Keep}_{c, l, d, p}=R E V_{c, l, d, p}+\text { Pregchk }_{p}
\end{aligned}
$$

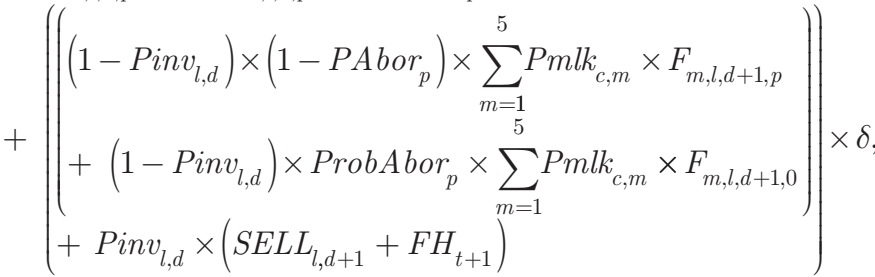

where Pregch $_{p}=$ cost of pregnancy diagnosis, and ProbAbor $_{p}=$ probability of abortion. The abortion term in equation [6] was excluded for cows with $<30$ DIP. The keep value of entering a cow to a new lactation was calculated by adding a constant calf value to equation [6].

4) Finally, if the cow was calving $(p=282)$ but was not in the last lactation $(l<9)$, then

$$
\begin{aligned}
& \text { Keep }_{c, l, d, 282}=R E V_{c, l, d, 282} \\
& +\left(\left(\begin{array}{l}
\left.\left(1-\operatorname{Pinv}_{l, d}\right) \times \sum_{m=1}^{5} \operatorname{Pmlk}_{c, m} \times F_{m, l+1,1,0}\right) \\
+\operatorname{Pinv}_{l, d} \times\left(S E L L_{l, d+1}+F H_{t+1}\right)
\end{array}\right) \times \delta .\right.
\end{aligned}
$$

The RPO for the current stage can be calculated based on the keep and replace values using the following equation:

$$
R P O_{c, l, d, p}=\operatorname{Keep}_{c, l, d, p}-\operatorname{Repl}_{c, l, d, p} .
$$

The RPO can be positive (when the keep value is higher than the replace value) or negative (when the replace value is higher than the keep value). The RPO can be used to rank cows for replacement decisions in a herd. A higher RPO represents a more valuable cow and an RPO below zero means culling of the cow is preferred.

\section{Markov Chain Model}

A daily Markov chain model (Giordano et al., 2012) was used to simulate dairy herd dynamics under studied reproductive performances. The Markov chain model found the herd structure at steady state or the proportion of cows at each defined state (De Vries, 2004; Cabrera, 2010; Kalantari et al., 2010). The Markov chain model resembled all the daily DP state dimensions, except the milk classes.

\section{Herd Value Calculation}

The herd value was defined as the herd's weighted average RPO, calculated as follows:

$$
\text { Herd value }=\sum_{l=1}^{9} \sum_{d=1}^{750} \sum_{p=0}^{282}\left(p_{l, d, p} \times R P O_{l, d, p}\right)
$$

where $p_{l, d, p}=$ proportion of cows in each state at steady state from the Markov chain model, and $R P O_{c, l, d, p}$ $=\mathrm{RPO}$ for each state determined by the DP model. This equation calculates the weighted average of RPO according to the proportion of available cows at each state. The herd value was calculated separately for each of the 5 different milk classes and for each of the defined reproductive programs.

\section{Computer Implementation}

The model was developed as a standalone executable program with Visual Basic Net 2010 (Microsoft Corp., Redmond, WA). Input variables were in the form of spreadsheet files and results were gathered as commaseparated files. 


\section{Model Parameters}

Milk Production. The incomplete gamma function (Wood, 1967) was used to estimate milk production throughout lactation. The Levenberg-Marquardt algorithm was used to minimize the difference between milk yield observations and estimated values. Factors of 5,10 , and $15 \%$ for milk production depression due to pregnancy were applied at 5, 6, and 7 mo in pregnancy, according to De Vries (2004). These monthly probabilities were converted to daily probabilities.

Milk Class Transition. The 15 milk classes as described by van Arendonk (1985) were merged to 5 milk classes. The fitted data were set to be an average milk class (third class) and other classes were set as a factor to this average milk class. For example, the lowest milk class was set to $76 \%$ of the third milk class and the highest milk class produced $124 \%$ of the third class. In van Arendonk's (1985) study, the repeatability of milk production was set to 0.55 monthly. Corresponding repeatability was empirically found to be 0.99 on a daily basis.

Carcass Value and Feed Intake. The daily BW for each state was calculated by the Korver function (Korver et al., 1985) as described by van Arendonk (1985). The model was parameterized to replicate the BW changes throughout lactation as shown in the NRC (2001) BW function. Body weight was also used to calculate the daily DMI. Dry matter intake was calculated according to NRC (2001) as a function of BW and $4 \%$ FCM.

Involuntary Culling and Reproductive Performance. The daily probability of involuntary culling for every cow state defined in the model was calculated based on the monthly involuntary culling described by De Vries et al. (2010).

A subset of 5 reproductive programs (RP) from Giordano et al. (2012) was studied (Table 1). Originally, 19 different RP were simulated in Giordano et al. (2012) to encompass plausible ranges of reproductive performance observed in commercial dairy farms. They compared the economic and reproductive performance of a program that used $100 \%$ timed AI (TAI) for all AI services with combined TAI plus estrus detection programs at different levels of estrus detection (Giordano et al., 2012). In these combined programs, the probability of insemination after estrus detection increased from 30 to $80 \%$ with 10-percentage-unit intervals at 3 conception rates of 25,30 , and $35 \%$ (Giordano et al., 2012). Therefore, each reproductive program was represented by a vector of daily pregnancy probabilities depending on their levels of estrus detection and conception rates. It has been shown that when estrus detection is added before or between TAI services, a conception rate reduction of those cows inseminated to TAI is observed (Chebel et al., 2004). A possible explanation of this reduction is that cows not detected in estrus and reaching TAI have lower fertility potential (Keskin et al., 2011). Thus, the baseline $40 \%$ conception rate after TAI at 30\% estrus detection in first service was decreased by 2 percentage units for each $10 \%$ increase in the estrus detection. For second and subsequent services, the conception rate was set at $30 \%$ when the estrus detection was between 30 and $50 \%$ and at $28 \%$ when estrus detection was between 60 and $80 \%$ (Giordano et al., 2012).

The $5 \mathrm{RP}$ studied here were selected to represent all the range of reproductive performances observed in Giordano et al. (2012): the 100\% TAI program and the combined programs at 2-percentage-unit intervals of 21-d pregnancy rates (21-d PR; Ferguson and Galligan, 1999). The first program used $100 \%$ TAI for all AI services and the other programs combined TAI with estrus detection with different levels of service and conception rates, (Table 1). The first program (RP1) used Presynch-Ovsynch for the first postpartum AI and Ovsynch for second and subsequent AI services with a 72-d VWP and an interbreeding interval of 42 d. Combined programs (RP2 to RP5) used the same synchronization protocol as RP1, except that AI after estrus detection was added between the end of VWP $(50 \mathrm{~d})$ and the first TAI at 72 DIM and in between TAI services (Giordano et al., 2012). These 5 RP were categorized according to their 21-d PR. The 21-d PR was $17 \%$ for RP1, $14 \%$ for RP2, $16 \%$ for RP3, $18 \%$ for $\mathrm{RP} 4$, and $20 \%$ for RP5.

Total daily reproductive cost for each program was calculated from the costs of labor for estrus detection and hormone injection and the costs of hormones for synchronization of ovulation and AI (Giordano et al., 2012). Additionally, constant costs related to pregnancy diagnosis, assuming rectal palpation, were applied at 39, 67, and 221 DIP. Pregnant cows had a daily probability of pregnancy loss from 30 DIP to term. These probabilities varied according to the stage of gestation. The probability of pregnancy loss was set at $0 \%$ for the first 30 DIP, $12.5 \%$ from 30 to 45 DIP, $9.9 \%$ from 46 to 180 DIP, and $2 \%$ from 181 DIP to term (Giordano et al., 2012).

Economic Parameters. Calves were assumed to be sold immediately, and the revenue from them was a weighted average price for male and female calves $(\$ 100)$. The yearly veterinary cost for an average firstlactation cow was set to $\$ 50$ and increased by $\$ 5$ each lactation (Groenendaal et al., 2004). These veterinary costs were assigned based on van Arendonk (1985): $33 \%$ to the first month of lactation, $11 \%$ to the second and third months in lactation, and the rest to the re- 
Table 1. Summary of studied reproductive programs

\begin{tabular}{|c|c|c|c|c|c|c|c|}
\hline $\begin{array}{l}\text { Reproductive } \\
\text { program }^{1}\end{array}$ & \multicolumn{3}{|c|}{ First AI } & \multicolumn{3}{|c|}{ Second and subsequent AI } & $\begin{array}{c}21-\mathrm{d} \\
\text { pregnancy } \\
\text { rate }(\%)\end{array}$ \\
\hline $\mathrm{RP} 2$ & 70 & 25 & 32 & 70 & 25 & 28 & 14 \\
\hline RP3 & 50 & 30 & 36 & 50 & 30 & 30 & 16 \\
\hline $\mathrm{RP} 4$ & 30 & 35 & 40 & 30 & 35 & 30 & 18 \\
\hline
\end{tabular}

${ }^{1}$ A subset of reproductive programs studied in Giordano et al. (2012). RP1 relied only on timed AI (TAI) for first AI with Presynch-Ovsynch protocol and for second and subsequent AI services with Ovsynch protocol, having a voluntary waiting period of $72 \mathrm{~d}$ and an interbreeding interval of $42 \mathrm{~d}$; RP2 to RP5 combined TAI with estrus detection between the end of the voluntary waiting period (50 d) and the first TAI at 72 DIM and during the subsequent re-synchronizations.

${ }^{2}$ Percentage of cows AI after estrus detection before TAI.

${ }^{3}$ Conception rate of cows AI after estrus detection before TAI.

mainder of the lactation. Other inputs are summarized in Table 2.

\section{RESULTS AND DISCUSSION}

\section{Herd Value Difference Between Reproductive Programs}

The herd values for $5 \mathrm{RP}$ across 5 different milk yields are presented in Table 3. Overall, results showed a positive relationship between $21-\mathrm{d} P R$ and herd value. At average milk yield, RP were ranked based on their herd values: RP5, RP4, RP1, RP3, and RP2 from highest to lowest. This ranking was consistent with that found with only the daily Markov chain model without milk classes (Giordano et al., 2012). However, Figure 1 reveals an interesting interaction between milk yield and RP. Every RP except RP5 showed some level of ranking change with relative milk yield. The RP5 with $20 \%$ 21-d PR was the absolute best program and did not show any ranking interaction with relative milk yield. The situation for the other analyzed RP was not stable across different milk yield classes. The most dramatic ranking change was observed for RP1 (100\% TAI) with $17 \%$ 21-d PR. This program's herd value changed from being the worst program at the lowest milk yield to the

Table 2. Economic parameters ${ }^{1}$

\begin{tabular}{lc}
\hline Price & Value \\
\hline Milk $(\$ / \mathrm{kg})$ & 0.36 \\
Calf value $(\$ /$ calf $)$ & 100 \\
Carcass value $(\$ / \mathrm{kg})$ & 1.16 \\
Replacement heifer cost $(\$)$ & 1,300 \\
Veterinary cost $(\$)$ & 50 \\
Feed cost in lactation $(\$ / \mathrm{kg})$ & 0.17 \\
Feed cost in dry period $(\$ / \mathrm{kg})$ & 0.13 \\
Annual interest rate $(\%)$ & 10 \\
\hline
\end{tabular}

${ }^{1}$ Same values as used in Giordano et al. (2012). second best program at highest milk yield. At the highest milk yield, RP4 (18\% 21-d PR) was ranked below RP1 (17\% 21-d PR).

The most extreme herd value difference $(\$ /$ cow per yr) between 2 reproductive programs was $\$ 77$ (RP5 RP2) for average milk yield, $\$ 13$ (RP5 - RP1) for $24 \%$ below average milk yield, and $\$ 160$ (RP5 - RP2) for $24 \%$ above average milk yield.

The large effect of relative milk yield on herd value is shown in Table 3 . The average herd value difference (\$/cow per yr) between the lowest and the highest milk production across all RP was $\$ 1,541$, and varied from $\$ 1,434$ (RP2) to $\$ 1,589$ (RP1). Main parameters affecting RPO in DP models have been well studied through sensitivity analysis (van Arendonk and Dijkhuizen, 1985; van Arendonk, 1985; Cardoso et al., 1999; Kalantari et al., 2010; Demeter et al., 2011). These studies have shown, using a monthly stage length DP, that the most important factors affecting RPO values are milk production, price of replacement heifer, and carcass value. The current study also found that the milk production is a very important factor determining the RPO.

The difference in herd values between RP is attributed to the effect of RP on the herd structure (percentage of milking and pregnant versus dry and open cows, and distribution of cows between and within lactations). Figure 2 shows the effect of different RP on the herd structure as well as on herd values. Figure 2 illustrates the RPO multiplied by the proportion of cows at each state across the first 3 lactations for 3 different RP (RP1, RP2, and RP5). Hence, Figure 2 is the daily RPO value weighted by the proportion of cows at each state (\$/cow per $\mathrm{d})$. In each lactation, the graph first shows a downward trend, which follows milk production curves. Before parturition, milk production is the main source of cow value, which is reflected by the milk production curve for the average RPO values. 
Table 3. Herd values (US\$) for 5 reproductive programs across 5 relative milk yields

\begin{tabular}{lccccc}
\hline & & \multicolumn{3}{c}{ Relative milk yield to average lactation curve (\%) } \\
\cline { 2 - 6 } $\begin{array}{l}\text { Reproductive } \\
\text { program }\end{array}$ & $\begin{array}{c}\text { 21-d pregnancy } \\
\text { rate (\%) }\end{array}$ & 76 & 88 & 100 & 112 \\
\hline RP1 & 17 & 156 & 374 & 769 & 1,224 \\
RP2 & 14 & 159 & 376 & 729 & 1,129 \\
RP3 & 16 & 161 & 385 & 763 & 1,190 \\
RP4 & 18 & 167 & 395 & 788 & 1,234 \\
RP5 & 20 & 169 & 410 & 806 & 1,593 \\
\hline
\end{tabular}

${ }^{1} \mathrm{RP} 1$ relied only on timed AI (TAI) for first AI with Presynch-Ovsynch protocol and for second and subsequent AI services with Ovsynch protocol, having a voluntary waiting period of $72 \mathrm{~d}$ and an interbreeding interval of $42 \mathrm{~d}$. RP2 to RP5 combined TAI with estrus detection between the end of the voluntary waiting period $(50 \mathrm{~d})$ and the first TAI at 72 DIM and during the subsequent re-synchronizations.

After reaching the nadir, which is a result of the RPO decreasing through lactation and proportion of cows during lactations, RPO shows an upward trend because of the expected value of a newborn calf. Following the first peak during each lactation, a steep, dented downward trend continues, which is a result of 2 factors. The first factor is parturition in a timely manner, based on the reproductive program characteristics. For example, RP1 shows a completely discrete pattern every $42 \mathrm{~d}$, reflecting its TAI interbreeding interval. The other 2 programs show more continuous patterns because of estrus detection breedings occurring in between TAI synchronizations.
The steep downward trend in Figure 2 is then the result of decreasing the proportion of cows throughout DIM along with lower RPO late in lactation. It is clear that the RP with lowest 21-d PR (RP2, 14\%) has the greatest value in first lactation. This illustrates that $\mathrm{RP}$ with lower 21-d PR have more reproductive and nonreproductive culling. Thus, these programs have a higher proportion of cows in first lactation and a lower proportion of cows in second and later lactations, which is less profitable. When comparing RP1 (100\% TAI) with RP5, it is clear that after the second lactation, RP1 falls behind the combined program (RP5). This can be mostly attributed to the high percentage of es-

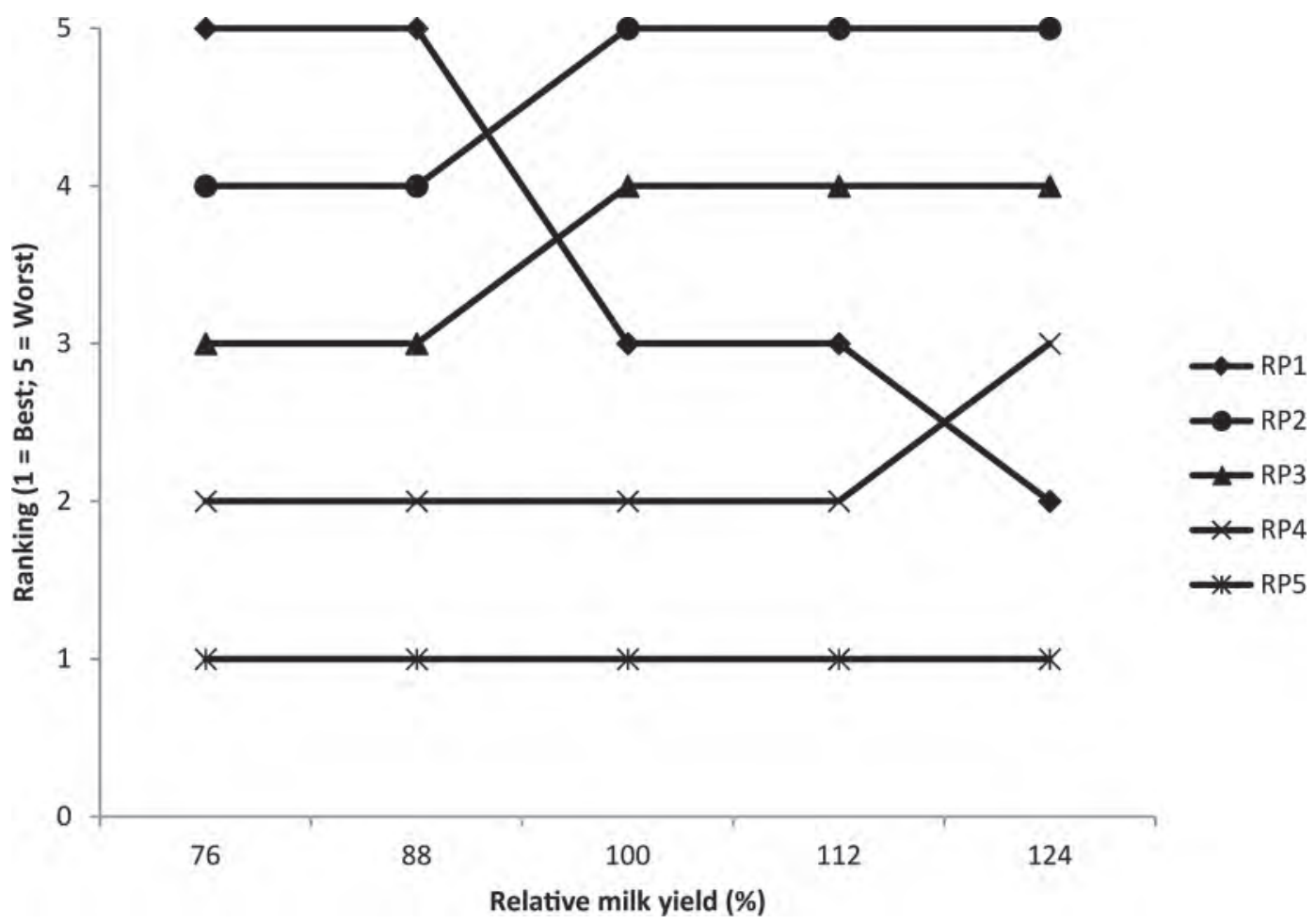

Figure 1. Ranking changes of 5 reproductive programs (RP) across 5 relative milk yields (\%). Reproductive program 1 relied only on timed $\mathrm{AI}(\mathrm{TAI})$ and had a 21-d pregnancy rate of 17\%; RP2 to RP5 combined TAI with estrus detection for 21-d pregnancy rates of 14, 16, 18, and $20 \%$, respectively. 


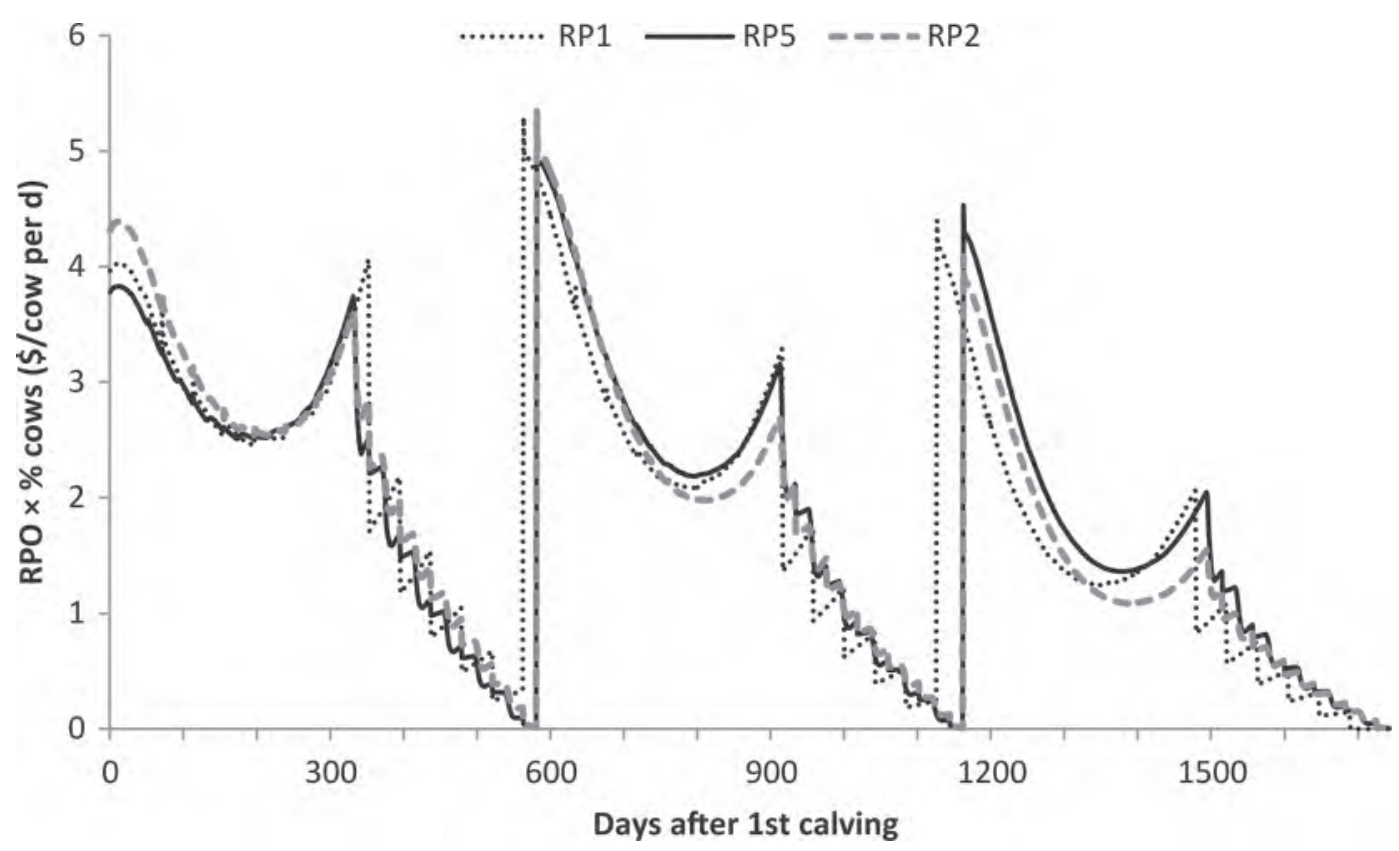

Figure 2. Product of retention payoff (RPO) by percentage of cows at each state in the first 3 lactations for 3 reproductive programs (RP). Reproductive program 1 relied only on timed AI (TAI) and had a 21-d pregnancy rate of 17\%; RP2 and RP5 combined TAI with estrus detection and had 14 and 20\% 21-d pregnancy rates, respectively.

trus detection (80\%) with a high conception rate (35\%) in the combined program (Table 1). Cows inseminated after estrous detection had a shorter interbreeding interval and greater conception rate than those cows reaching the TAI. (Giordano et al., 2012). These differences in conception rate and estrus detection were reflected in a 3-percentage-unit difference in 21-d PR (17 vs. 20\%) between RP1 and RP5.

\section{RPO Difference Within an RP}

Figure 3 shows the effect of DIM and pregnancy time on the RPO. The open cow RPO follows a milk curve pattern. The cow value is the highest at the beginning and gradually decreases through lactation. The unusually greater open RPO on the first day in lactation is a result of adding the value of the newborn calf on this first day. This greater open RPO is obscured in monthly DP models (De Vries, 2006; Kalantari et al., 2010). The pregnant cow RPO curve, which is equal to the difference between the RPO of pregnant and nonpregnant cow at the same DIM, changes based on the time in pregnancy. With increasing DIM at pregnancy, pregnancy value curves (pregnant RPO value) start closer to the RPO of open cows. The pregnant RPO value of a cow becoming pregnant at 200 DIM for second and later lactations was less than the RPO of open cows. For first-lactation cows, this occurred at about 260 DIM (data not shown). However, the final
RPO of a pregnant cow at parturition was similar in spite of DIM at pregnancy (about $\$ 1,450$ ). The lowest RPO value in the daily DP model developed in this study was $-\$ 10$, which is much less than that found with a monthly model ( $-\$ 184$; Kalantari et al., 2010). A much lower RPO negative value was also found by the daily DP model of Nielsen et al. (2010). Daily RPO is the opportunity cost of keeping a cow for only one more day, and therefore a negative value does not accumulate for longer periods.

The trends on RPO and the relative values are more important than the actual calculated values (Groenendaal et al., 2004). Therefore, panel A of Figure 4 is presented to illustrate the RPO trend throughout 9 lactations for different DIM at pregnancy. It is clear that increasing DIM at pregnancy from 55 to $200 \mathrm{~d}$ changes the shape of the curves slightly. The maximum RPO occurred in the fourth lactation and after that, it decreased consistently until the ninth lactation, which was the maximum possible cow life. Similar trends were previously reported in monthly models (Groenendaal et al., 2004; Kalantari et al., 2010). Generally, the RPO of cows decreased after parturition until the point of pregnancy. After pregnancy, the RPO showed a large jump to either a higher or lower value (depending on the DIM at pregnancy; Figure 4, panel A). The times of a pregnancy in first and second lactations were obvious: a vertical upward straight line can be seen. However, these lines were obscured after second lactation. Dur- 


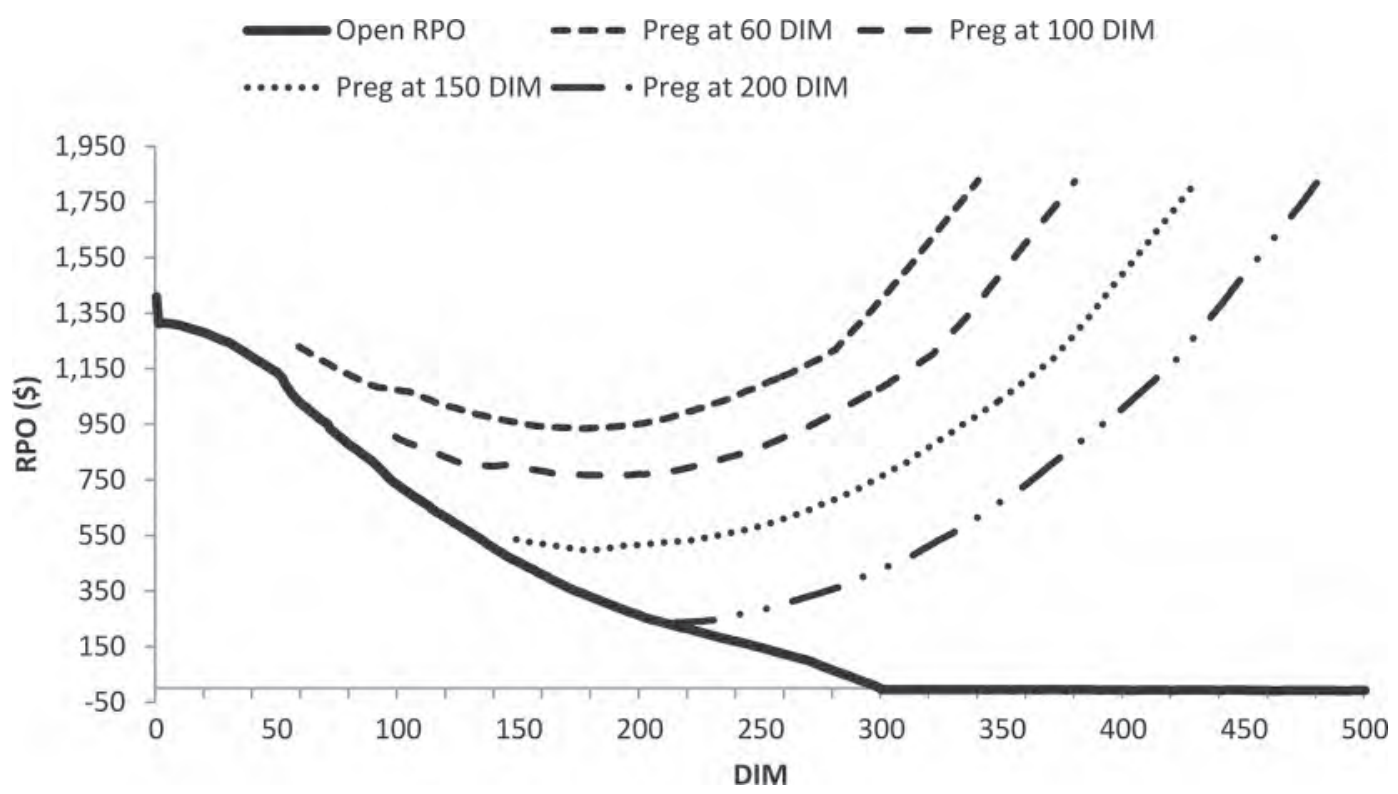

Figure 3. Retention payoff (RPO) values for cows at different DIM of pregnancy in the second lactation for the average milk class and reproductive program RP5 (which combined timed AI with estrus detection and had a 21-d pregnancy rate of 20\%).

ing early pregnancy, the RPO usually showed a slight decrease in value. This decrease depended on the lactation and DIM at which the cow was pregnant. The RPO of a cow pregnant at 55 DIM decreased slightly in each lactation and then increased until calving. One difference when pregnancy occurred at 200 DIM was that the RPO increased permanently after pregnancy until the time of calving (panel A in Figure 4; Figure 3 ). This difference in trend of the RPO in pregnant cows between early and late pregnancy was mainly a result of the effect of expected milk production (determined by the projected milk production curve) and involuntary culling on the net revenue. That is, cows becoming pregnant late in lactation will have a smaller difference between keep and replace values than cows becoming pregnant early in the lactation. This differential in value is because of events occurring in late lactation: a decrease in natural milk production and increase in involuntary culling, either or both of which may occur. As a result, a higher value is placed on getting cows pregnant late in late lactation. This translates to a permanent increase of RPO of pregnant cows late in lactation (Figure 3; Figure 4). Despite early or late pregnancy, the RPO of pregnant cows increased to parturition time because of the expected value of the calf for the next lactation (Figure 4, panels A and B).

To compare the overall value of cows becoming pregnant at different DIM within a defined reproductive program (RP5), the accumulated weighted RPO for each cow state over a cow's lifetime were calculated. Accumulated weighted RPO value for a cow becoming pregnant at 55 DIM was $\$ 409$ and for a cow becoming pregnant at 200 DIM was $\$ 333$, a $\$ 76$ difference between these 2 scenarios. This difference was a result of RPO changes only throughout lactation because the herd structure remained the same for these 2 scenarios. The RPO for cows becoming pregnant later in the lactation was always lower (De Vries, 2004).

The RPO values for the first 3 lactations for RP5, average milk class, and 120 DIM at conception are shown in Figure 4, panel B. This shows the effect of pregnancy loss on RPO at different DIM comparing 2 scenarios of cows having pregnancy loss with one scenario of a cow without a pregnancy loss: one cow has a pregnancy loss at 170 DIM (50 DIP) and is successfully rebred 30 d later; another cow has a pregnancy loss at 220 DIM (100 DIP) and is successfully rebred $30 \mathrm{~d}$ later. The $\mathrm{RPO}$ of the cows losing a pregnancy decreased dramatically until the next successful conception. This decrease in RPO depended on the DIP at which the pregnancy loss happened. As in the previous case, the effect of pregnancy loss on the overall value was calculated as the weighted average RPO (RPO times the proportion of cows at that specific state) throughout 9 lactations. This value for the cow without a pregnancy loss was $\$ 370, \$ 29$ greater than the cow with pregnancy loss at 50 DIP (and successful rebred 30 d later) and $\$ 36$ greater than the cow with pregnancy loss at 100 DIP (and successful rebred $30 \mathrm{~d}$ later). It is obvious that pregnancy loss had a considerable effect on the overall RPO of cows in the herd, even though a successful rebreeding occurred soon after. 


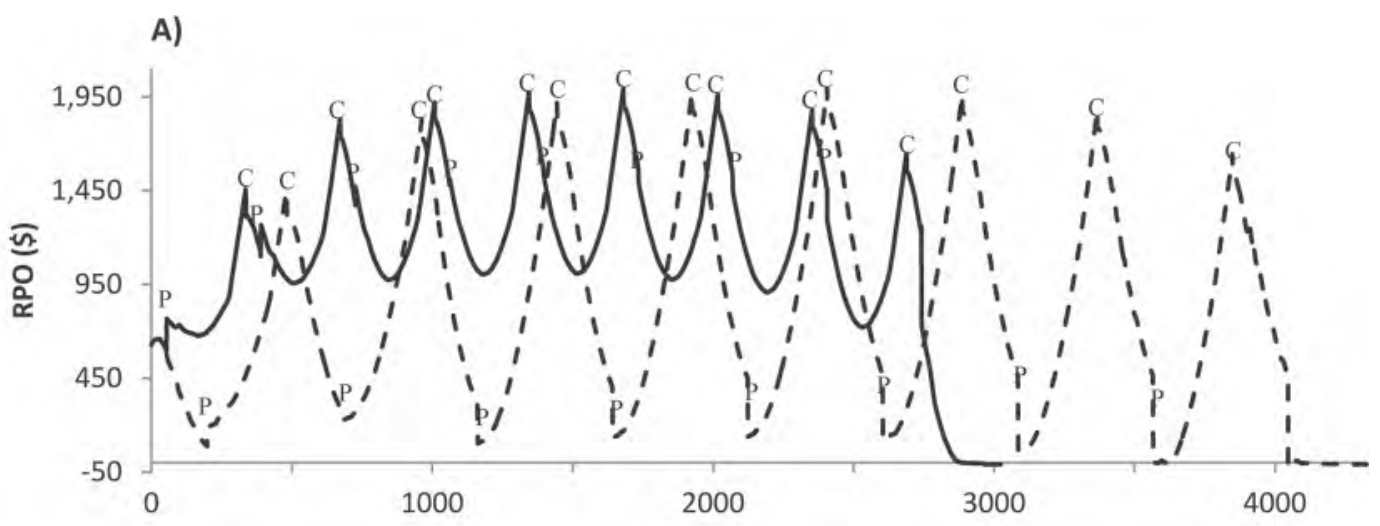

B)

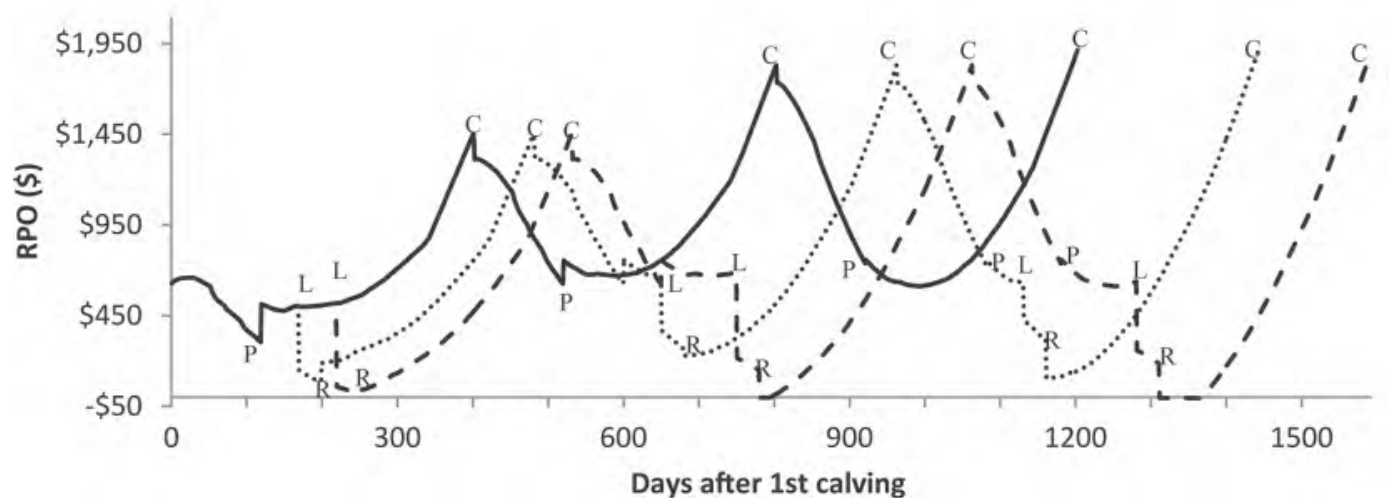

Figure 4. Daily retention payoff (RPO) of reproductive program 5 (which combined timed AI with estrus detection with 21-d pregnancy rate of 20\%) and average milk class under different scenarios. (A) Pregnancy at 55 DIM (-), pregnancy at 200 DIM (---) during 9 lactations; (B) pregnancy at 120 DIM without pregnancy loss (-), pregnancy at 120 DIM with pregnancy loss at 170 DIM and successfully rebred at 200 DIM (...), and pregnancy at 120 DIM with pregnancy loss at 220 DIM and successfully rebred at 250 DIM (- - - ) during each of the first 3 lactations. Labels show events: pregnancy $(\mathrm{P})$, pregnancy loss $(\mathrm{L})$, successfully rebred $(\mathrm{R})$, and calving $(\mathrm{C})$.

\section{Implication for Dairy Farm Decision-Making and Management}

Our results support the notion that an opportunity exists to adjust reproductive programs according to milk classes and therefore according to RPO. The modeling framework could be used for daily decisions of assigning cows to different reproductive management groups based on their RPO. This would promote more cost-effective reproductive programs and therefore overall improved herd value. In addition, results demonstrate an interaction among herd value, relative milk yield, and reproductive programs at different levels of estrus detection and TAI. Therefore, daily reproductive decisions might include, for example, whether to breed a heat-detected cow or not. Cows with higher relative milk yield would benefit more from TAI reproductive programs, whereas cows with lower relative milk yield would be better off with estrus detection programs (Figure 1).
Replacement decisions are being made daily on dairy farms and these decisions have a great effect on the herd profitability. Despite their great impact on profitability, these decisions are still made arbitrarily. The most important aspect of the DP model is its ability to rank cows in the herd based on their value. This ranking could be an important guideline to replace the least profitable animals in the herd. Dairy farmers, extension professionals, and farm advisers could take advantage of this information to help decision-making and management on dairy farms. Indeed, the models presented here could be incorporated into software already being used on dairy farms. The current DP model's daily stage length has the potential to provide more accurate $\mathrm{RPO}$ and to be used as a guideline in these crucial daily decisions. Moreover, the ranking could be used in conjunction with reproductive decisions, such as distinguishing the quality and type of semen according to daily RPO ranking. A possible refinement of the 
DP model could include the decision whether or not to breed eligible cows on a daily basis.

The DP model could be modified to address a greater range of dairy farms such as organic or grazing farms. For example, those farms having a goal to promote the longevity of milk cows could use suboptimal decisions: this could be addressed by multiplying the keep value in the objective function (Eq. [1]) by a constant factor $(\alpha)$. Our default formulation assumes an $\alpha$ value of 1. Farms willing to keep cows for longer than their economically optimal lifetime would use an empirical valuation of this factor $>1$. On the opposite end of the spectrum, farms wanting a faster turnover of animals because of potential herd genetic gain would have this value be $<1$. The decisions with this modification could be, to some extent, subjective, but could accommodate particular farm objectives.

An important advantage of using daily stage in the models presented in this study is the ability to better represent farm-specific and detailed reproductive management strategies. For example, the models could be adjusted to farms that use natural service by modifying the probability of pregnancy calculations. Because the actual time of insemination is not known, stochastic distribution could be used to simulate possible observed patterns. Therefore, distributions could be applied to simulate the unknown services times. Currently, a similar methodology is implemented to simulate the distribution of cows showing estrus. Although DP optimal decisions are not sensitive to the probability of pregnancy (van Arendonk and Dijkhuizen, 1985), the daily Markov chain model is highly dependent on these probabilities. Additionally, the daily Markov chain model could be used to analyze the cost effectiveness of different pregnancy diagnosis techniques and their interaction with reproductive programs.

Another application of the DP model could be in veterinary treatment decisions (Demeter et al., 2011). Positive RPO represents the expected value of keeping a cow one more day, and negative RPO is the opportunity cost of keeping this cow one more day. A positive RPO could be interpreted as the maximum acceptable treatment cost that could be spent in treating a cow (van Arendonk, 1988). On the other hand, a negative RPO shows the amount of money the farm is losing by keeping the cow one more day. Indeed, the daily DP model could be used by researchers to estimate more accurately the costs of days open, new pregnancy values, or the value of pregnancy losses. Ultimately, both models (daily DP and daily Markov chain) could be integrated with existing models to perform wholefarm studies.

As with any simulation and optimization model, the current models have some limitations. The models presented here do not include health problems, such as mastitis and lameness, or seasonality, which have been shown to have considerable effect on optimal decisions, herd structure, and herd net return (Houben et al., 1994; De Vries, 2004; Cha et al., 2010). These limitations could be overcome by including more state variables. However, inclusion of new states makes the state space of the models grow exponentially. Large DP models could become unsolvable. Hence, a tradeoff exists between including more state variables and decreasing stage length.

\section{CONCLUSIONS}

A daily DP model was developed to evaluate the effect of different reproductive programs on herd value when coupled with a daily Markov chain model. Results showed that herd values were largely influenced by reproductive programs. In addition, an interaction was observed among the herd value, milk yields, and reproductive programs. Results support the notion that reproductive programs or specific reproductive events could be designed according to the individual cow expected production level for improved herd value. Within the same reproductive program, the RPO changed based on the stage of lactation at pregnancy. Cows becoming pregnant early in lactation had greater RPO than cows becoming pregnant later in lactation.

\section{ACKNOWLEDGMENTS}

This project was supported by Agriculture and Food Research Initiative Competitive Grant no. 2010-8512220612 from the USDA National Institute of Food and Agriculture (Washington, DC). This research was also supported by Hatch project (WIS01577) to V.E.C.

\section{REFERENCES}

Britt, J. H. 1985. Enhanced reproduction and its economic implications. J. Dairy Sci. 68:1585-1592.

Cabrera, V. E. 2010. A large Markovian linear program to optimize replacement policies and dairy herd net income for diets and nitrogen excretion. J. Dairy Sci. 93:394-406.

Cabrera, V. E., and J. O. Giordano. 2010. Economic decision making for reproduction. Pages 77-86 in Proc. Dairy Cattle Reproduction Council Convention, St. Paul, MN. Dairy Cattle Reproduction Council, Hartland, WI.

Cardoso, V. L., J. R. Nogueira, and J. A. M. van Arendonk. 1999. Optimal replacement and insemination policies for Holstein cattle in the southeastern region of Brazil: The effect of selling animals for production. J. Dairy Sci. 82:1449-1458.

Cha, E., J. A. Hertl, D. Bar, and Y. T. Gröhn. 2010. The cost of different types of lameness in dairy cows calculated by dynamic programming. Prev. Vet. Med. 97:1-8.

Chebel, R. C., J. E. Santos, J. P. Reynolds, R. L. Cerri, S. O. Juchem, and M. Overton. 2004. Factors affecting conception rate after artificial insemination and pregnancy loss in lactating dairy cows. Anim. Reprod. Sci. 84:239-255. 
De Vries, A. 2004. Economics of delayed replacement when cow performance is seasonal. J. Dairy Sci. 87:2947-2958.

De Vries, A. 2006. Economic value of pregnancy in dairy cattle. J. Dairy Sci. 89:3876-3885.

De Vries, A., J. D. Olson, and P. J. Pinedo. 2010. Reproductive risk factors for culling and productive life in large dairy herds in the eastern United States between 2001 and 2006. J. Dairy Sci. 93:613-623.

Demeter, R. M., A. R. Kristensen, J. Dijkstra, A. G. J. M. Oude Lansink, M. P. M. Meuwissen, and J. A. M. van Arendonk. 2011. A multi-level hierarchic Markov process with Bayesian updating for herd optimization and simulation in dairy cattle. J. Dairy Sci. 94:5938-5962.

Ferguson, J. D., and D. T. Galligan. 1999. Veterinary reproductive programs. Pages 131-137 in Proc. 32nd Annual Convention of the American Association of Bovine Practitioners, Nashville, TN. AABP, Auburn, AL.

Giordano, J. O., P. M. Fricke, M. C. Wiltbank, and V. E. Cabrera 2011a. An economic decision-making support system for selection of reproductive management programs on dairy farms. J. Dairy Sci. 94:6216-6232.

Giordano, J. O., P. M. Fricke, M. C. Wiltbank, and V. E. Cabrera 2011b. Daily Markov-chain simulation model for selection of reproductive management programs in dairy herds. J. Anim. Sci 89(E-Suppl. 1):256. (Abstr.)

Giordano, J. O., A. S. Kalantari, P. M. Fricke, M. C. Wiltbank, and V. E. Cabrera. 2012. A daily herd Markov-chain model to study the reproductive and economic impact of reproductive programs combining timed artificial insemination and estrus detection. J. Dairy Sci. 95:5442-5460.

Groenendaal, H., D. T. Galligan, and H. A. Mulder. 2004. An economic spreadsheet model to determine optimal breeding and replacement decisions for dairy cattle. J. Dairy Sci. 87:2146-2157.

Houben, E. H. P., R. B. M. Huirne, A. A. Dijkhuizen, and A. R. Kristensen. 1994. Optimal replacement of mastitic cows determined by a hierarchic Markov process. J. Dairy Sci. 77:2975-2993.

Kalantari, A. S., H. Mehrabani-Yeganeh, M. Moradi, A. H. Sanders, and A. De Vries. 2010. Determining the optimum replacement policy for Holstein dairy herds in Iran. J. Dairy Sci. 93:2262-2270.
Keskin, A., G. Yilmazbas-Mecitoglu, E. Karakaya, A. Alkan, H. Okut, A. Gumen, and M. C. Wiltbank. 2011. Effect of presynchronization strategy prior to Ovsynch on fertility at first service in lactating dairy cows. J. Dairy Sci. 94(E-Suppl. 1):191. (Abstr.)

Korver, S., J. A. M. van Arendonk, and W. J. Koops. 1985. A function for live weight change between two calvings in dairy cattle. Anim. Prod. 40:233-241.

Kristensen, A. R. 1988. Hierarchic Markov processes and their applications in replacement models. Eur. J. Oper. Res. 35:207-215.

Meadows, C., P. J. Rajala-Schultz, and G. S. Frazer. 2005. A spreadsheet-based model demonstrating the nonuniform economic effects of varying reproductive performance in Ohio dairy herds. J. Dairy Sci. 88:1244-1254.

Nielsen, L. R., E. Jørgensen, A. R. Kristensen, and S. Ostergaard. 2010. Optimal replacement policies for dairy cows based on daily yield measurements. J. Dairy Sci. 93:75-92.

NRC. 2001. Nutrient Requirements of Dairy Cattle. 7th ed. Natl. Acad. Press, Washington, DC.

Olynk, N. J., and C. A. Wolf. 2008. Economic analysis of reproductive management strategies on US commercial dairy farms. J. Dairy Sci. 91:4082-4091

Olynk, N. J., and C. A. Wolf. 2009. Stochastic economic analysis of dairy cattle artificial insemination reproductive management programs. J. Dairy Sci. 92:1290-1299.

Smith, B. J. 1973. Dynamic programming of the dairy cow replacement problem. Am. J. Agric. Econ. 55:100-104.

van Arendonk, J. A. M. 1985. Studies on the replacement policies in dairy cattle. II. Optimum policy and influence of changes in production and prices. Livest. Prod. Sci. 13:101-121.

van Arendonk, J. A. M. 1988. Management guides for insemination and replacement decisions. J. Dairy Sci. 71:1050-1057.

van Arendonk, J. A. M., and A. A. Dijkhuizen. 1985. Studies on the replacement policies in dairy cattle. III. Influence of variation in reproduction and production. Livest. Prod. Sci. 13:333-349.

Wood, P. D. P. 1967. Algebraic model of the lactation curve in cattle. Nature 216:164-165 